\title{
The importance of lipoxygenase control in the production of green leaf volatiles by lipase-dependent and independent pathways
}

\author{
Cynthia Mugo Mwenda, Kenji Matsui* \\ Graduate School of Medicine and Department of Biological Chemistry, Faculty of Agriculture, Yamaguchi University, \\ Yamaguchi 753-8515, Japan \\ *E-mail: matsui@yamaguchi-u.ac.jp Tel: +81-83-933-5850 Fax: +81-83-933-5820
}

Received August 28, 2014; accepted September 24, 2014 (Edited by T. Shoji)

\begin{abstract}
Green leaf volatiles (GLVs) are six-carbon volatile compounds. They are formed from fatty acids by a dioxygenation reaction catalyzed by lipoxygenase and a subsequent cleavage reaction catalyzed by hydroperoxide lyase. GLVs are involved in direct and indirect plant defense against herbivores and pathogens. In intact plant tissues, GLVs are usually present at low concentrations, but upon wounding, GLVs are synthesized rapidly: within seconds to minutes. It has been hypothesized that this 'GLV burst' is supported by activation of pre-existing enzymes on endogenous substrates; however, the detailed mechanism of the GLV burst has not been elucidated. Recently, we found that a certain portion of GLVs is formed without liberation of free fatty acids from lipids. Accordingly, we hypothesized that lipoxygenase plays an essential role in the GLV burst. In particular, direct oxygenation by lipoxygenase on membrane lipids seems to be responsible. Lipoxygenase is also a target for controlling GLV levels in food derived from plants.
\end{abstract}

Key words: Green leaf volatile, hydroperoxide lyase, lipase, lipoxygenase.

\section{Introduction}

Green leaf volatile (GLV) is a generic name referring to six-carbon aldehydes, alcohols and their esters. 2-Hexenal was first isolated from green leaves of hornbeam maple (Acer carpinifolium) in 1912 (Curtius and Franzen, 1912). Thereafter, chemists in Kyoto and Yamaguchi Universities in Japan largely clarified the full range of GLVs (Hatanaka, 1993) (Figure 1). These early efforts were performed mainly to identify the origin of green leaf-like odors found in most plant leaves and in foods or beverages, such as green tea. Almost all land plants, including bryophytes, ferns, gymnosperms, and angiosperms, can produce GLVs (Hatanaka 1993). Even marine organisms, e.g., brown alga, Laminaria angustata, have the ability (Boonprab et al. 2003). It has been proposed that the gene for GLV formation, hydroperoxide lyase (HPL), was acquired in the last common ancestor of plants and animals. Even though HPL-related genes are found in Trichoplax, corals, hydra, and sea anemone, and in bacteria such as Methylobacterium strains, most animals do not have the genes and thus cannot form GLVs. The HPL-related genes seem to have been lost during evolution of the animal lineage (Lee et al. 2008).

The most important signature of GLVs is their rapid formation after disruption of plant tissues, the 'GLV burst.' In general, intact and healthy plants contain relatively low levels of GLVs; however, mechanical wounding of plant tissues, especially on leaves, instantly induces the formation of GLVs. In mechanically wounded Arabidopsis leaves, formation of (Z)-3-hexenal was evident within seconds and reached its maximum level at $30-45 \mathrm{~s}$ following damage. The emission of its reduced form, (Z)-3-hexen-1-ol, followed (peaking at $2.5 \mathrm{~min}$ ), and subsequently $(Z)-3$-hexen-1-yl acetate followed (peaking at approximately $5 \mathrm{~min}$ ) (D'Auria et al. 2007). This 'GLV burst' is considered to be associated with responses to biotic and abiotic stresses related to tissue injury, such as herbivore damage or infection of necrotrophic pathogens. Recently, a comprehensive review was published on the recent progress in elucidating the physiological and ecological significance of GLVs (Scala et al. 2013). In this review, we focus on recent advances in clarifying the mechanism that accomplishes the GLV burst upon tissue disruption. 


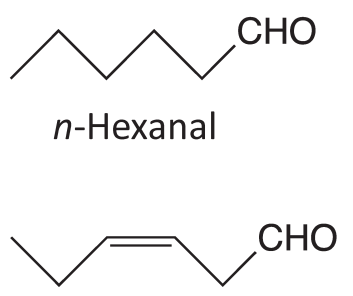

(Z)-3-Hexenal

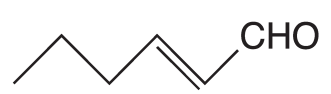

(E)-2-Hexenal<smiles>CCCCCCO</smiles><smiles>CC/C=C\CCO</smiles>

(Z)-3-Hexen-1-ol

(Green leaf alcohol)<smiles>CCC/C=C/CO</smiles>

(E)-2-Hexen-1-ol

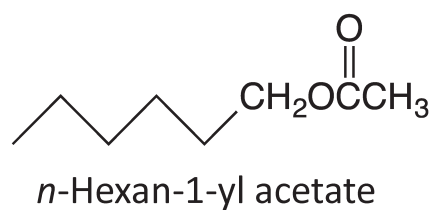<smiles>CC/C=C\CCCOC(C)=O</smiles>

(Z)-3-Hexen-1-yl acetate<smiles>CCC/C=C/COC(C)=O</smiles>

(E)-2-Hexen-1-yl acetate

(Green leaf aldehyde)

Figure 1. Structures of typical green leaf volatiles. (E)-2-Hexenal and (Z)-3-hexen-1-ol have generic names, as shown in parentheses. In some plants, $(E)$-3-isomers of their $(Z)$-3-counterparts are found. In addition, the esters with butanoates and hexanoates are also formed in some plant species.

GLVs are also important flavor compounds for wide varieties of plant-derived foods, fresh vegetables and fruits. Recently, several studies have indicated that molecular breeding techniques could be applied to control the GLV contents in crops to manipulate their flavor properties. This issue is also briefly summarized here.

\section{GLVs are oxylipins derived from lipids}

In most living organisms, lipids are not only components of membranes and energy storage compounds, but are also precursors of various bioactive molecules. In animal cells, arachidonic acid is an especially important fatty acid precursor for a wide array of lipid-mediators, such as prostaglandins and leukotrienes (Shimizu and Wolfe 1990). Plant cells also form a wide array of bioactive compounds from fatty acids, including GLVs. Jasmonic acid and its derivatives (jasmonates, JAs), which show diverse physiological activities during growth, development and plant defense (Wasternack and Hause 2013), are also formed from fatty acids. The compounds derived from an oxidation reaction of fatty acids, such as GLVs or JAs, are collectively termed plant oxylipins (Mosblech et al. 2009). LOX (lipoxygenase) seems to be the enzyme that diverts the primary metabolites (fatty acids, in this case) to the secondary metabolites leading to oxylipins in plants. LOX inserts molecular oxygen into the 9- or 13-position of carbon 18 polyunsaturated fatty acids, such as linoleic and linolenic acids. The hydroperoxides thus formed serve as substrates for HPLs that lead to GLVs, or for allene oxide synthases (AOSs) that lead to JAs (Figure 2).

\section{A lipase-dependent pathway}

Most plant LOXs prefer free fatty acids as substrates, at least in vitro (Ivanov et al. 2010); therefore, it has been assumed that liberation of free fatty acids from esterified lipids must be a preceding event in the formation of oxylipins. Much research has been devoted to identifying the lipases involved in oxylipin synthesis through liberation of linoleic and linolenic acids from lipids. DAD1 was reported as a phospholipase A1 that is essential to JA formation in the stamen filaments of Arabidopsis flowers (Ishiguro et al. 2001). However, DAD1 might not be the lipase delivering free fatty acids to the JA pathway in wound-induced JA formation in leaves (Wasternack and Hause 2013). Ellinger et al. (2010) reported that DAD1 and DONGLE (encoded by one of the DAD1 isologs in Arabidopsis) were not essential for JA formation in mechanically wounded Arabidopsis leaves. On the contrary, they reported that PLA1 $\gamma 1$ (encoded by another isolog of DAD1 in Arabidopsis), was at least partly involved in woundinduced JA accumulation (Ellinger et al. 2010). GLYCEROLIPASE A1 (GLA1) in Nicotiana attenuata is thought to be a lipase involved in JA biosynthesis during herbivorous and mechanical wounding in leaves, but not during Phytophthora parasitica infection (Bonaventure et al. 2011). Accordingly, it is assumed that there must be stimuli-specific lipases that are responsible for JA formation (Wasternack and Hause 2013).

The involvement of lipases in GLV formation in plants has not been confirmed. Apart from the case of JAs, where a free carboxylic acid moiety in their biosynthetic intermediate, 12-oxo phytodienoic acid (OPDA), is essential for $\beta$-oxidation in peroxisomes, GLV can be 


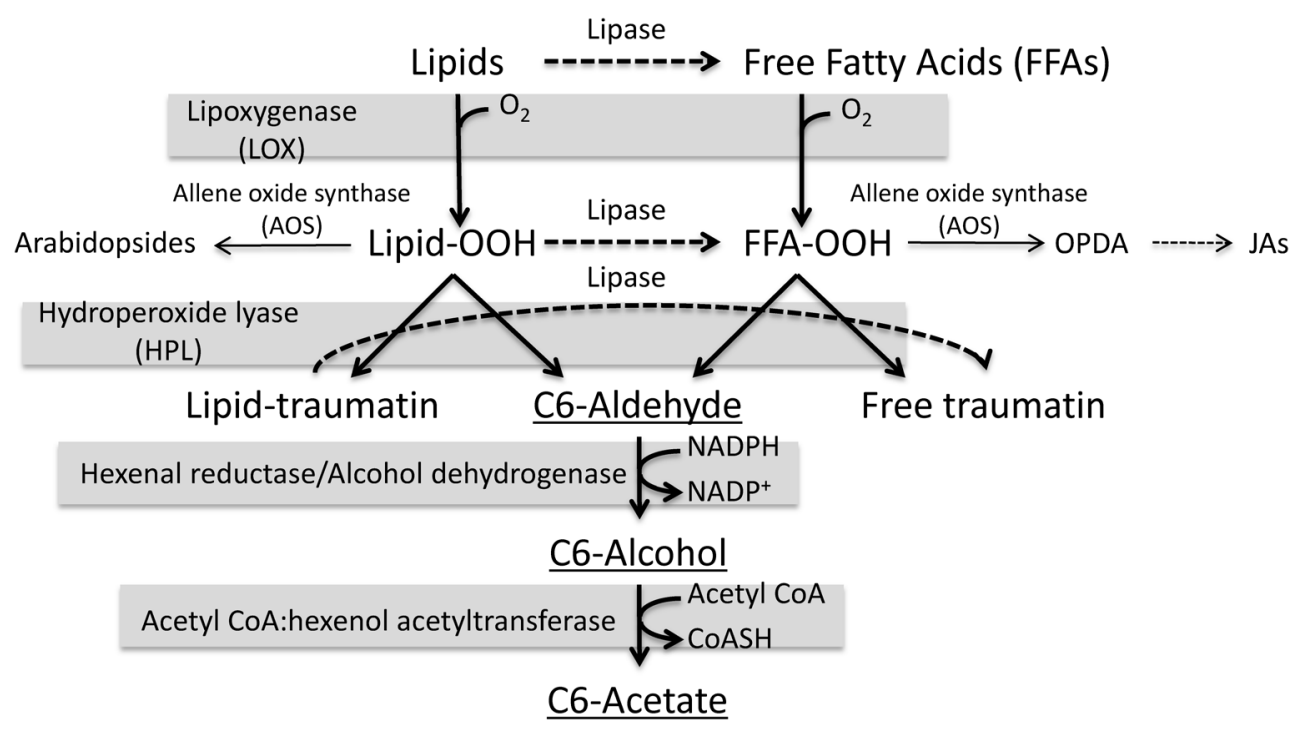

Figure 2. Schematic drawing of the two major oxylipin pathways (GLV and JA pathways) in plants, with emphasis on the GLV pathway.

formed either as free fatty acids or esterified fatty acids (Figure 2). When Arabidopsis leaves were disrupted, formation of 12-carbon (C12) oxo acids [12-oxo-(Z)-9dodecenoic acid (traumatin), and its derivatives], which are counterparts of the HPL reaction, was observed in their free forms, albeit at levels that were substantially lower than those expected from the amounts of GLVs formed (Nakashima et al. 2013). Formation of traumatin and its derivatives in mechanically wounded Nicotiana attenuata leaves was also reported (Kallenbach et al. 2011). Therefore, an involvement of lipases in the formation of GLVs is suggested; however, it is uncertain whether the lipase action occurs before or after the LOX reaction, or even after the LOX/HPL reactions on lipids. Addition of $\left[1-{ }^{14} \mathrm{C}\right]$ linolenic acid into chloroplasts partially purified from leaves of tea resulted in the formation of a labeled traumatin-derivative (Hatanaka et al. 1977). Therefore, free fatty acids, once they are formed in plant tissues, could be converted enzymatically to GLVs. Mutant dad1 Arabidopsis formed GLVs after disruption of leaves at the equivalent level found with wild-type Arabidopsis. Transgenic N. attenuata with reduced expression of GLA1 showed almost the same level of GLV burst after mechanical wounding as wildtype plants (Bonaventure et al. 2011).

Collectively, it is assumed that a lipase is involved, at least partly, in the pathway that forms GLVs from lipids; however, it is still uncertain which lipid substrates the lipase would hydrolyze. There are three possible substrates: unmodified lipids to form free fatty acids, lipid hydroperoxides to form fatty acid hydroperoxides, and lipids containing traumatins as their acyl group to form traumatins (Figure 2). Lipids are highly hydrophobic, and it is difficult to set up an in vitro reaction condition to monitor their activities. In addition, some lipases need cofactors, such as co- lipases, or a specified oil/water interface for their optimal reaction (Aloulou et al. 2006). It is assumed that lipases involved in GLV formation, as well as those involved in the other bioactive plant oxylipins, play a critical role in controlling their production; therefore, detailed knowledge on these lipases is essential. Further investigation is needed to identify a specific lipase for each biosynthetic step and to clarify its properties.

\section{A lipase-independent pathway}

Mono- and digalactosyldiacylglycerols (MGDG and DGDG) that contain OPDA and/or 12oxodinorphytodienoic acid (a 16-carbon analog of OPDA) (collectively known as arabidopsides) have been identified in Arabidopsis (Buseman et al. 2006; Stelmach et al. 2001) (Figure 3). Phospholipids and galactolipids containing divinyl ether residues (linolipins) (Figure 3) have also been found in potato tubers and flax seedlings, respectively (Chechetkin et al. 2009; Fauconnier et al. 2003). Identification of these lipid-bound oxylipins implies that oxygenation and subsequent modification of the acyl groups of the lipids may proceed without the liberation of free fatty acid by a lipase action.

When Arabidopsis leaves were disrupted, GLVs formed instantly (within seconds) and their amounts reached

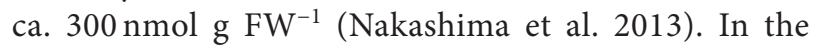
disrupted Arabidopsis leaves, the counterpart of products of the HPL reaction, namely 12-oxo-(Z)-9-dodecenoic acid [(9Z)-traumatin] and its derivatives, such as 12-oxo(E)-10-dodecenoic acid [(10E)-traumatin] and 12-oxo9-hydroxy-(E)-10-dodecenoic acid (9-OH-traumatin), should be formed in a stoichiometric manner. However, the total amounts of these 12-carbon (C12) oxo acids reached only $10-20 \mathrm{nmol} \mathrm{g} \mathrm{FW}^{-1}$. 'Dinor' (C10) forms of them (presumably formed from hexadecatrienoic 

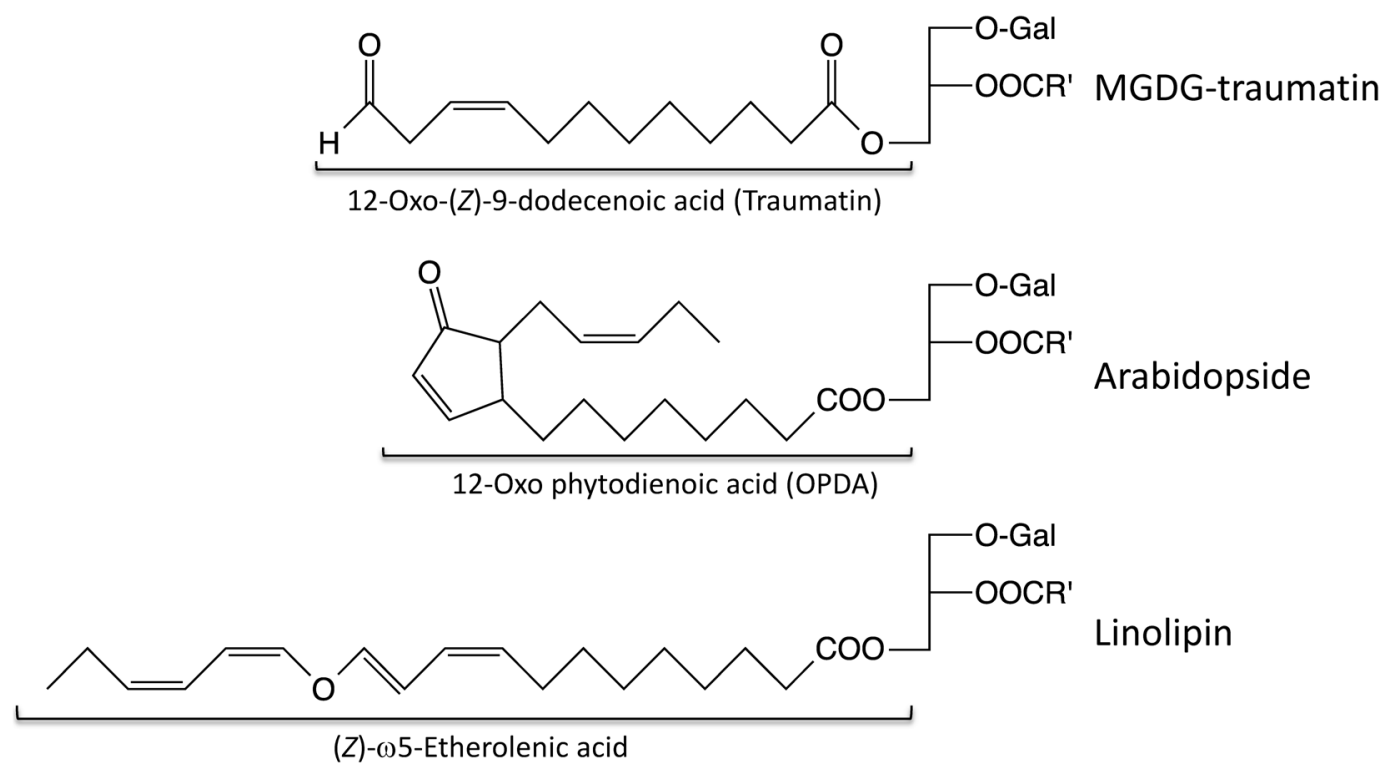

Figure 3. Structures of representative lipid oxylipins. MGDG traumatin is formed from the HPL pathway. In Arabidopsis and Nicotiana attenuata, traumatin is further modified through isomerization of the double bond to the (E)-10-form and/or oxygenation at the C9 position. The oxylipin with OPDA is formed from the AOS pathway, and the related compounds are collectively named arabidopsides. In flax, MGDG with an acyl moiety containing divinyl ether structure is found, and its related compounds are named linolipins. In either case, the oxylipin can be found at either the $s n 1$ or $s n 2$ position, or both. These lipid oxylipins might also occur with DGDG, or with phospholipids, such as phosphatidylcholine. R indicates the glycerol backbone. R' indicates an acyl group. Gal stands for galactose.

acid, which is abundant in galactolipids of C16:3 plants including Arabidopsis (Li-Beisson et al. 2010) were also formed, but at an equally low level to their C12 analogs. Instead, reaction of crude lipids extracted from disrupted leaves with pancreatin (containing both phospholipase and galactolipase activities) yielded substantial amounts of the $\mathrm{C} 12$ compounds in their free forms. This indicated that traumatins existed as esters bound on lipids. Accordingly, a proportion of GLVs must be formed without a lipase reaction on lipids. Extensive liquid chromatography tandem mass spectrometry (LC-MS/ MS) analyses indicated an accumulation of galactolipids containing $\mathrm{C} 12$ compounds as their acyl groups. One of the esterified oxylipins was purified and its structure was determined as $O$-[9-hydroxy-12-oxo- $(E)-10$ dodecenyl]-O-[7-hydroxy-10-oxo-(E)-8-decenyl]-O- $\beta$-Dgalactopyranosyl-glycerol (MGDG-9-OH-traumatin/7$\mathrm{OH}$-dinortraumatin) through nuclear magnetic resonance and high-resolution MS analyses (Nakashima et al. 2013). LC-MS/MS analysis suggested that several other compounds structurally related to MGDG-9$\mathrm{OH}$-traumatin/7-OH-dinortraumatin were also formed during disruption of Arabidopsis. An Arabidopsis mutant lacking active HPL activity formed only trace amounts of galactolipids with traumatin and/or dinortraumatin derivatives as their acyl groups: therefore, their formation was dependent on active HPL.

This indicated that GLVs are formed partially in a lipase-independent manner directly from galactolipids. Formation of galactolipids with $\mathrm{C} 12 / \mathrm{C} 10$ oxo acids was detected in disrupted leaves of cabbages, tobacco, tomatoes, and beans; thus, the lipase-independent pathway might be widespread in the plant kingdom. In the lipase-independent GLV pathway, LOX should act on galactolipids. Soybean LOX1 showed little activity on purified MGDG; however, addition of an appropriate detergent, such as deoxycholic acid, significantly increased its activity on MGDG (Nakashima et al. 2011). The effect of deoxycholic acid was concentrationdependent, and deoxycholic acid at $7 \mathrm{mM}$ showed the most potent effect; the detergent was less effective at lower or higher concentrations. This implied that soybean LOX1 needs certain water/lipid interface conditions to show its activity. This was also the case with the catalytic activities of soybean LOX1 and cucumber root LOX on a positively charged phospholipid, phosphatidylcholine (Eskola and Laakso 1983; Matsui et al. 1998). In the disrupted plant tissues, the architectures of organelle membranes, including the thylakoid membrane, which contains vast amounts of galactolipids, would be distorted (Figure 4). This change of membrane architecture might be a prerequisite for LOX to catalyze its oxygenation reaction on lipids, probably through interfacial activation, which has been observed for lipases (Aloulou et al. 2006). Thereafter, HPL, which is bound on the chloroplast envelope (Froehlich et al. 2001), attaches on the MGDG-OOH to convert it to MGDG traumatin and hexenal. With the direct reaction of LOX on lipids, GLVs could be formed more quickly than with the lipasedependent pathway, which would meet the requirement for plants to exert their wound response as quickly as possible. 


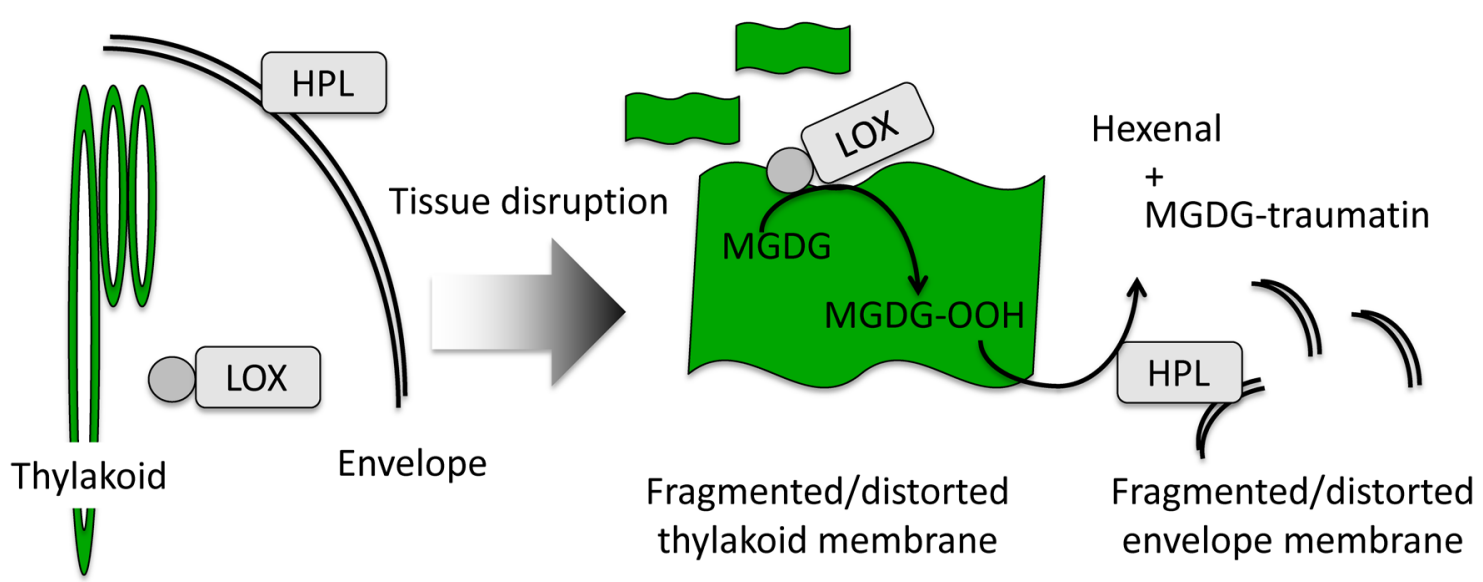

Figure 4. A proposed model for the GLV burst triggered by tissue disruption. TomLOXC (through GFP analysis) (Chen et al. 2004) and NaLOX2 (through TargetP prediction) (Allman et al. 2010) were predicted to be located in the stroma of chloroplasts. Tomato HPL was predicted to bind on the outer envelope membrane of chloroplasts (Froehlich et al. 2001). Upon disruption of tissues, chloroplasts would collapse, and thylakoid membranes (rich in MGDG) would be fragmented. Even though most plant LOXs are soluble enzymes, they have N-terminal $\beta$-barrel domains that are assumed to interact with a hydrophobic surface (Ivanov et al. 2010), which would help LOXs to interact with the fragmented thylakoid membrane to catalyze formation of MGDG-OOH from MGDG. At the same time, the envelope membrane would be fragmented, allowing HPL bound on it to access MGDG-OOH. ZmLOX10 was found in organelles other than chloroplasts, and collapse of the organelles would result in the same activation.

Even though rapid formation of GLVs after mechanical wounding of plant leaves has long been recognized, the precise mechanism supporting the 'GLV burst' has not been elucidated. The discovery of the lipase-independent GLV pathway might be a clue to the mechanism, because it is assumed that the LOX reaction on lipids is a key step in controlling the formation of GLVs. However, there are still many issues to be resolved. For example, most plants have several to a dozen LOX isoforms. A specific LOX isoform essential for GLV formation has been identified only in a few plant species, e.g., ZmLOX10 in maize (Christensen et al. 2013), TomloxC in tomato (Chen et al. 2004), or lipoxygenase 2 in Nicotiana attenuata (Allman et al. 2010), among others. The specific LOX isoform must first be identified and its subcellular localization determined. Second, we should clarify the mechanism for how LOX is activated biochemically upon tissue disruption. Most LOXs are soluble enzymes; therefore, the hydrophobic interaction of LOXs with their substrates (lipids in membrane) might be regulated by an as yet unknown mechanism. Furthermore, the reason why most plants have both the lipase-dependent and independent GLV pathways, and whether these two pathways are differentiated, remains unknown. Last, the biological activities of esterified oxylipin have been reported in mammals, e.g., the truncated, terminally oxidized phospholipids were proposed as ligands for the macrophage scavenger receptor CD36 (Podrez et al. 2002). Galactolipids with $\mathrm{C} 12 / \mathrm{C} 10$ oxo acids might also have biological activities on plants or humans when the disrupted plant materials are consumed, which must be investigated in a future study.

\section{Further modification of GLVs}

A portion of the $(Z)$-3-hexenal formed by HPL is immediately reduced to (Z)-3-hexen-1-ol. Subsequently, (Z)-3-hexen-1-ol is partly converted into $(Z)$-3-hexen1 -yl acetate (D’Auria et al. 2007). In some cases, (Z)-3hexenal is isomerized to $(E)$-2-hexenal (Allmann and Baldwin 2010), which is subsequently converted into its alcohol- and acetate-forms. The rates of isomerization, reduction, and acetylation, and also the proportion of (Z)-3-hexenal to be converted further, differ greatly among plant species; therefore, the composition of GLVs differs extensively among plant species. The differences in the activity of each converting step also result in time- and type of wound (cause)-dependent changes in the amount and composition of GLVs. Thus, it can be assumed that the compositions of GLVs bear additional information related to time and cause in organism (such as herbivores or carnivores) that perceive GLVs as infochemicals (Allmann and Baldwin 2010).

Alcohol dehydrogenase (ADH) was assumed to catalyze the reduction of C6 aldehydes into alcohols (Hatanaka 1993). When ADH activity was modulated through genetic manipulation of the tomato $A D H$ gene, the levels of C6 alcohols roughly correlated with the ADH activities in the transgenic tomatoes (Speirs et al. 1998). By contrast, a tomato short-chain ADH (SlscADH1) showed high activity with $n$-hexanal and (E)-2-hexenal, but little activity with (Z)-3-hexenal, even though $(Z)$-3-hexen-1-ol is one of the most abundant GLVs in tomatoes (Moummou et al. 2012). An Arabidopsis mutant lacking ADH activity showed 10 -fold higher amounts of hexenal than that of wildtype; however, the mutant still showed a substantial 
ability to form C6 alcohol (Bate et al. 1998). These results indicated that $\mathrm{ADH}$ is involved in the formation of $\mathrm{C} 6$ alcohols only in part or indirectly, and that there must be other enzyme systems employed to reduce hexenals. When Arabidopsis leaves were disrupted in the presence of NADPH, the hexenol/hexenal ratio was significantly increased, while the aldehyde/alcohol ratio with the other volatiles scarcely changed (Matsui et al. 2012). The addition of NADH showed a much weaker effect. This implied that there must be an NADPH-specific aldo-keto reductase with a relatively strict substrate specificity to C6 aldehydes. In Arabidopsis, several enzymes belonging to the aldo-keto reductase superfamily or NADB Rossmann (short-chain dehydrogenase/reductase) superfamily are involved in detoxification of reactive carbonyls having $\alpha, \beta$-unsaturated carbonyl moieties, such as (E)-2-hexenal (Yamauchi et al. 2011). Again, it is uncertain whether the Arabidopsis reductases examined so far can reduce ( $Z$ )-3-hexenal to $(Z)$-3-hexen-1-ol. The identification of the reductase essential to $(Z)$-3-hexen-1ol formation is still an open question.

Arabidopsis has an acetyl CoA:(Z)-3-hexen-1-ol acetyltransferase with high reactivity to (Z)-3-hexen-1-ol (D’Auria et al. 2007). By contrast, strawberries' alcohol acyltransferase (FaAAT2) shows substrate specificity to saturated short-chain alcohol, and (Z)-3-hexen-1-ol was not acetylated (Cumplido-Laso et al. 2012). Again, there must be diverse acyltransferases in the plant kingdom with different substrate/product specificities, which cause diversity in GLV composition among plant species or even among plant organs in the same species. Tomatoes have a carboxyesterase (SICXE1) that catalyzes conversion of (Z)-3-hexen-1-yl acetate to (Z)-3-hexen1-ol (Goulet et al. 2012), which is also detrimental to the composition of GLV blends; however, it is not clear whether a similar enzyme is detrimental to GLV compositions in other plants.

It has been assumed that the main function of GLV formation is to defend against enemies (pathogens or herbivores) who attempt to invade through the wounds on plant tissues. In this context, formation of C6aldehydes is reasonable because it intrinsically harbors relatively high reactivity as an acceptor of the Schiff base formation reaction with various biological molecules. (E)-2-Hexenal, formed from (Z)-3-hexenal through isomerization, is more promising because of its reactivity as an acceptor of the Michael addition reaction, owing to the $\alpha, \beta$-unsaturated carbonyl moiety. Even (Z)-3-hexenal is a potentially toxic compound to the invaders because it is prone to be oxygenized, either enzymatically by LOX or spontaneously, to yield 4-hydroxy- and 4-oxo-(E)-2hexenal (Matsui et al. 2012). All these functions rely on an aldehyde moiety; however, C6-alcohols and acetates do not have the functional group, which raises the question of why most plants reduce and acetylate reactive carbonyl species.

When Arabidopsis seedlings were exposed to vapor of (Z)-3- or (E)-2-hexenal, their photosynthetic activities rapidly dropped, and after several hours, the rims of leaves showed necrotic lesions (Matsui et al. 2012). Thus, the hexenals are themselves toxic to plants. We postulated that the original reason why plants acquired a system to reduce and acetylate C6-aldehydes is to avoid their toxic effect. In some plant species, the C6alcohols and/or C6-acetate are beneficial to producers because they attract carnivores that attack the herbivores feeding on the producers. In this case, the reduction and acetylation capabilities would further enhance the fitness of the producers.

\section{Molecular breeding techniques to control the GLV contents of crops}

Whether GLVs would be preferable as flavor constituents depends on the nature of the food in which they are found. They are preferred constituents in most fresh fruits, such as tomatoes, apples and pears (Mayer et al. 2008, Song and Forney 2008, Souleyre et al. 2014), and in olive oil (Kalua et al. 2007). For foods made from soybeans, they can provide either a good or off flavor. For tofu (soybean curd), GLVs are essential to add thickness to the flavor (Matsui et al. 2011), while for soymilk, they are responsible for an off flavor. LOX is a prerequisite for GLV formation, as well as the other flavor compounds related to oxidized or rancid fat (such as five- and eightcarbon volatiles); therefore, LOX has been the main target to regulate the amount of GLVs. In soybean seeds there are three LOXs, all of which contribute to the formation of GLVs in homogenized soybean seeds (i.e., in soymilk) (Kobayashi et al. 1995). To obtain a soybean variety that produced soymilk with low GLV contents, Hajika and coworkers bred a variety that lacked all three LOXs through a classic breeding technique concomitant with mutagenesis induced by $\gamma$-irradiation (Hajika et al. 1991). Soymilk made from the LOX-less variety contained significantly lower amounts of GLVs, and had a less 'beany' flavor (Torres-Penaranda et al. 1998). A similar result was obtained in rice seeds that lacked one of the LOXs found in rice embryos (LOX3): they had lower stale flavor properties derived from hexanal, pentanal and pentan-1-ol (Suzuki et al. 1999).

Whole genome sequencing of important crops and polymerase chain reaction-based genotyping useful to quantitative trait locus (QTL) analyses has led to further advances in molecular breeding to control GLV contents of crops. In apples, QTLs for each component of GLVs were located at several chromosomal loci (Vogt et al. 2013). Comparing the QTL maps for GLV components and LOX genes on the chromosomes revealed a substantial correlation between them, which might be a 
useful tool to breed a new apple variety that has better flavor properties through molecular breeding strategies (Vogt et al. 2013). This QTL-based approach also resulted in confirmation of a locus (alcohol acetyl transferase 1: AAT1) that contributes the 'ripe apple' flavor in apples (Souleyre et al. 2014). Thus, the QTL-based molecular breeding technique is available to produce new varieties of crops with appropriate GLV contents that are preferred by consumers.

\section{Acknowledgements}

This work was supported partly by JSPS KAKENHI Grant Number 23580151 and 26660095, and the Yobimizu Project from Yamaguchi University.

\section{References}

Allmann S, Baldwin IT (2010) Insects betray themselves in nature to predators by rapid isomerization of green leaf volatiles. Science 329: 1075-1078

Allmann S, Halitschke R, Schuurink R, Baldwin IT (2010) Oxylipin channeling in Nicotiana attenuate: Lipoxygenase 2 supplies substrates for green leaf volatile production. Plant Cell Environ 33: $2028-2040$

Aloulou A, Rodriguez JA, Fernandez S, van Oosterhout D, Puccineli D, Carriére F (2006) Exploring the specific features of interfacial enzymology based on lipase studies. Biochim Biophys Acta 1761: 995-1013

Bate NJ, Riley JCM, Thompson JE, Rothstein SJ (1998) Quantitative and qualitative differences in $\mathrm{C}_{6}$-volatile production from the lipoxygenase pathway in an alcohol dehydrogenase mutant of Arabidopsis thaliana. Physiol Plant 104: 97-104

Boonprab K, Matsui K, Akakabe Y, Yotsukura N, Kajiwara T (2003) Hydroperoxy-arachidonic acid mediated $n$-hexanal and (Z)-3- and (E)-2-nonenal formation in Laminaria angustata. Phytochemistry 63: 669-678

Bonaventure G, Schuck S, Baldwin IT (2011) Revealing complexity and specificity in the activation of lipase-mediated oxylipin biosynthesis: A specific role of the Nicotiana attenuata GLA1 lipase in the activation of jasmonic acid biosynthesis in leaves and roots. Plant Cell Environ 34: 1507-1520

Buseman CM, Tamura P, Sparks AA, Baughman EJ, Maatta S, Zhao J, Roth MR, Esch SW, Shah J, Williams TD, et al. (2006) Wounding stimulates the accumulation of glycerolipids containing oxophytodienoic acid and dinor-oxophytodienoic acid in Arabidopsis leaves. Plant Physiol 142: 28-39

Chechetkin IR, Mukhitova FK, Blufard AS, Yarin AY, Antsygina LL, Grechkin AN (2009) Unprecedented pathogen-inducible complex oxylipins from flax-linolipins A and B. FEBS J 276: 4463-4472

Chen G, Hackett R, Walker D, Taylor A, Lin Z, Grierson D (2004) Identification of a specific isoform of tomato lipoxygenase (TomloxC) involved in the generation of fatty acid-derived flavor compounds. Plant Physiol 136: 2641-2651

Christensen SA, Nemchenko A, Borrego E, Murray I, Sobhy IS, Bosak L, DeBlasio S, Erb M, Robert CAM, Vaughn KA, et al. (2013) The maize lipoxygenase, $Z m L O X 10$, mediates green leaf volatile, jasmonate and herbivore-induced plant volatile production for defense against insect attack. Plant J 74: 59-73

Cumplido-Laso G, Medina-Puche L, Moyano E, Hoffmann T,
Sinz Q, Ring L, Studart-Wittkowski C, Caballero JL, Schqab W, Muñoz-Blanco J, et al. (2012) The fruit ripening-related gene FaAAT2 encodes an acyl transferase involved in strawberry aroma biogenesis. J Exp Bot 63: 4275-4290

Curtius T, Franzen H (1912) Über die chemischen Bestandteile grüner Pflanzen. Über den Blätteraldehyd. 89-121

D’Auria JC, Pichersky E, Schaub A, Hansel A, Gershenzon J (2007) Characterization of BAHD acyltransferase responsible for producing the green leaf volatile (Z)-3-hexen-1-yl acetate in Arabidopsis thaliana. Plant J 49: 194-207

Ellinger D, Stingl N, Kubigsteltig II, Bals T, Juenger M, Pollmann S, Berger S, Schuenemann D, Mueller MJ (2010) DONGLE and DEFECTIVE IN ANTHER DEHISCENCE1 lipases are not essential for wound- and pathogen-induced jasmonate biosynthesis: Redundant lipase contribute to jasmonate formation. Plant Physiol 153: 114-127

Eskola K, Laakso S (1983) Bile salt-dependent oxygenation of polyunsaturated phosphatidylcholines by soybean lipoxygenase-1. Biochim Biophys Acta 751: 305-311

Fauconnier ML, Williams TD, Marlier M, Wleti R (2003) Potato tuber phospholipids contain colneleic acid in the 2-position. FEBS Lett 538: 155-158

Froehlich JE, Itoh A, Howe GA (2001) Tomato allene oxide synthase and fatty acid hydroperoxide lyase, two cytochrome P450s involved in oxylipin metabolism, are targeted to different membranes of chloroplast envelope. Plant Physiol 125: 306-317

Goulet C, Mageroy MH, Lam NB, Floystad A, Tieman DM, Klee HJ (2012) Role of an esterase in flavor volatile variation within the tomato clade. Proc Natl Acad Sci USA 109: 19009-19014

Hajika M, Igita K, Kitamura K (1991) A line lacking all the seed lipoxygenase isozymes in soybean [Glycine max (L.) Merrill] induced by gamma-ray irradiation. Jpn J Breed 41: 507-509

Hatanaka A (1993) The biogeneration of green odour by green leaves. Phytochemistry 34: 1201-1218

Hatanaka A, Kajiwara T, Sekiya J, Kido Y (1977) Formation of 12-oxo-trans-10-dodecenoic acid in chloroplasts from Thea sinensis leaves. Phytochemistry 16: 1828-1829

Ishiguro S, Kawai-Oda A, Ueda J, Nishida I, Okada K (2001) The DEFECTIVE IN ANTHER DEHISCENCE1 gene encodes a novel phospholipase A1 catalyzing the initial step of jasmonic acid biosynthesis, which synchronized pollen maturation. Plant Cell 13: 2191-2209

Ivanov I, Heydeck D, Hofheinz K, Roffeis J, O’Donnell VB, Kuhn H, Walther M (2010) Molecular enzymology of lipoxygenases. Arch Biochem Biophys 503: 161-174

Kallenbach M, Gilardoni PA, Allmann S, Baldwin IT, Bonaventure $\mathrm{G}$ (2011) $\mathrm{C}_{12}$ derivatives of the hydroperoxide lyase pathway are produced by product recycling through lipoxygenase- 2 in Nicotiana attenuata leaves. New Phytol 191: 1054-1068

Kalua CM, Allen MS, Bedgood DR Jr, Bishop AG, Prenzler PD, Robards K (2007) Olive oil volatile compounds, flavour development and quality: A critical review. Food Chem 100: 273-286

Kobayashi A, Tsuda Y, Hirata N, Kubota K, Kitamura K (1995) Aroma constituents of soybean [Glycin max (L.) Merril] milk lacking lipoxygenase isozymes. J Agric Food Chem 43: 2449-2452

Lee DS, Nioche P, Hamberg M, Raman CS (2008) Structural insights into the evolutionary paths of oxylipin biosynthetic enzymes. Nature 455: 363-368

Li-Beisson Y, Shorrosh B, Beisson F, Andersson MX, Arondel V, Bates PD, Baud S, Bird D, Debono A, Durrett TP, et al. (2010) Acyl-lipid Metabolism. Arabidopsis Book 8: e0133 
Matsui K, Nishioka M, Ikeyoshi M, Matsumura Y, Mori T, Kajiwara $\mathrm{T}$ (1998) Cucumber root lipoxygenase can act on acyl groups in phosphatidylcholine. Biochim Biophys Acta 1390: 8-20

Matsui K, Takaki S, Shimada K, Hajika M (2011) Effects of anaerobic processing of soybean seeds on the properties of tofu. Biosci Biotechnol Biochem 75: 1174-1176

Matsui K, Sugimoto K, Mano J, Ozawa R, Takabayashi J (2012) Differential metabolisms of green leaf volatiles in injured and intact parts of a wounded leaf meet distinct ecophysiological requirements. PLoS ONE 7: e36433

Mayer F, Takeoka GR, Buttery RG, Whitehand LC, Naim M, Rabinowitch HD (2008) Studies on the aroma of five fresh tomato cultivars and the precursors of cis- and trans-4,5-epoxy(E)-2-decenals and methional. J Agric Food Chem 56: 3749-3754

Mosblech A, Feussner I, Heilmann I (2009) Oxylipins: Structurally diverse metablotites from fatty acid oxidation. Plant Physiol Biochem 47: 511-517

Moummou H, Tonfack LB, Cervin C, Benichou M, Youmbi E, Ginies C, Latché A, Pech JC, van der Rest B (2012) Functional characterization of SlscADH1, a fruit-ripening-associated shortchain alcohol dehydrogenase of tomato. J Plant Physiol 169: 1435-1444

Nakashima A, Iijima Y, Aoki K, Shibata D, Sugimoto K, Takabayashi J, Matsui K (2011) Monogalactosyl diacylglycerol is a substrate for lipoxygenase: Its implications for oxylipin formation directly from lipids. J Plant Interact 6: 93-97

Nakashima A, von Reuss SH, Tasaka H, Nomura M, Mochizuki S, Iijima Y, Aoki K, Shibata D, Boland W, Takabayashi J, et al. (2013) Traumatin- and dinortraumatin-containing galactolipids in Arabidopsis: Their formation in tissue-disrupted leaves as counterparts of green leaf volatiles. J Biol Chem 288: 26078-26088

Podrez EA, Poliakov E, Shen Z, Zhang R, Deng Y, Sun M, Finton PJ, Shan L, Febbraio M, Hajjar DP, et al. (2002) A novel family of atherogenic oxidized phospholipids promotes macrophage foam cell formation via the scavenger receptor CD36 and is enriched in atherosclerotic lesions. J Biol Chem 277: 38517-38523

Scala A, Allmann S, Mirabella R, Haring MA, Schuurink RC (2013) Green leaf volatiles: A plant's multifunctional weapon against herbivores and pathogens. Int J Mol Sci 14: 17781-17911

Shimizu T, Wolfe LS (1990) Arachidonic acid cascade and signal transduction. J Neurochem 55: 1-15

Song J, Forney CF (2008) Flavour volatile production and regulation in fruit. Can J Plant Sci 88: 537-550

Souleyre EJF, Chagné D, Chen X, Tomes S, Turner RM, Wang MY, Maddumage R, Hunt MB, Winz RA, Wiedow C, et al. (2014) The AAT1 locus is critical for the biosynthesis of esters contributing to 'ripe apple' flavor in 'Royal Gala' and 'Granny Smith' apples. Plant J 78: 903-915

Speirs J, Lee E, Holt K, Young-Duk K, Scott NS, Loveys B, Schuch W (1998) Genetic manipulation of alcohol dehydrogenase levels in ripening tomato fruit affects the balance of some flavor aldehydes and alcohols. Plant Physiol 117: 1047-1058

Stelmach BA, Müller A, Hennig P, Gebhardt S, Schubert-Zsilavecz M, Weiler EW (2001) A novel class of oxylipins, sn1-O-(12oxophytodienoyl)-sn2-O-(hexadecatrienoyl)-monogalactosyl diglyceride, from Arabidopsis thaliana. J Biol Chem 276: 12832-12838

Suzuki Y, Ise K, Li C, Honda I, Iwai Y, Matsukura U (1999) Volatile components in stored rice [Oryza sativa (L.)] of varieties with and without lipoxygenase-3 in seeds. J Agric Food Chem 47: 1119-1124

Torres-Penaranda AV, Reitmeier CA, Wilson LA, Fehr WR, Narvel JM (1998) Sensory characteristics of soymilk and tofu made from lipoxygenase-free and normal soybeans. J Food Sci 63: 1084-1087

Vogt J, Schiller D, Ulrich D, Schwab W, Dunemann F (2013) Identification of lipoxygenase (LOX) genes putatively involved in fruit flavor formation in apple (Malus $\times$ domestica). Tree Genet Genomes 9: 1493-1511

Wasternack C, Hause B (2013) Jasmonates: Biosynthesis, perception, signal transduction and action in plant stress response, growth and development. An update to the 2007 review in Annals of Botany. Ann Bot (Lond) 111: 1021-1058

Yamauchi Y, Hasegawa A, Taninaka A, Mizutani M, Sugimoto Y (2011) NADPH-dependent reductases involved in the detoxification of reactive carbonyls in plants. J Biol Chem 286: 6999-7009 